\title{
Electric field bridging pattern of pre-breakdown and breakdown condition in transformer oil
}

\author{
Nur Badariah Ahmad Mustafa ${ }^{1}$, N H Nik Ali ${ }^{2}$, H. Zainuddin ${ }^{3}$, Marizuana Mat Daud ${ }^{4}$, \\ Farah Hani Nordin ${ }^{5}$ \\ ${ }^{1,5}$ Department of Electrical and Electronics Engineering, College of Engineering, Universiti Tenaga Nasional, Malaysia \\ ${ }^{2}$ Institute of Power Engineering, Universiti Tenaga Nasional, Malaysia \\ ${ }^{3}$ Faculty of Electrical Engineering, Universiti Teknikal Malaysia Melaka (UTeM), Malaysia \\ ${ }^{4}$ MIMOS Berhad, Technology Park Malaysia, Malaysia
}

\begin{tabular}{l} 
Article Info \\
\hline Article history: \\
Received Jan 2, 2020 \\
Revised Mar 29, 2020 \\
Accepted Apr 17, 2020 \\
\hline Keywords: \\
Breakdown \\
Electric field bridging \\
Pattern recognition \\
Pre-breakdown \\
Transformer oil
\end{tabular}

\begin{abstract}
Transformer is considered as one of the most important equipment in electrical power system networks. However, most problems occurred in transformer were related to the defects and weakness of the insulation systems. The oils used in transformer act as coolant and insulation purposes hence maintaining the dielectric strength of the transformer. In this work, electric field bridging pattern is observed from pre-breakdown and breakdown condition. The electric field bridging formation was recorded in the experimental setup and images were captured per frame. 193 images were randomly chosen from the whole video frames where 102 images were the pre-breakdown images and 91 images were the breakdown images. This system comprises of four stages: (i) a preprocessing stage to mark the electrodes tips and background subtraction; (ii) a segmentation stage to extract the electric field bridging formation in region of interest; (iii) a feature extraction stage to extract electric field bridging using feature descriptors, area, minor-axis and major-axis length (iv) a classification stage to identify the pre-breakdown and breakdown condition. System performance was evaluated using support vector machine (SVM), $k$-nearest neighbour $(k-\mathrm{NN})$ and random forest (RF) and SVM provided the most promising accuracy that was $99 \%$. The results show that the combination of three feature descriptors, area, minor-axis and major-axis length are the best features combination in identifying the transformer oil condition. In future work, further studies will be conducted to investigate the pattern of pre- and post-breakdown due to some similarity found in image pattern. Due to that, more feature descriptors will be identified to find a unique pattern between pre- and post-breakdown condition.
\end{abstract}

Copyright $@ 2020$ Institute of Advanced Engineering and Science. All rights reserved.

\section{Corresponding Author:}

Nur Badariah Ahmad Mustafa,

Department of Electrical and Electronics,

College of Engineering,

Universiti Tenaga Nasional, Malaysia.

Email: NBadariah@uniten.edu.my

\section{INTRODUCTION}

Transformer oil or insulating oil is a type of oil that is stable at high temperatures and has excellent electrical insulating properties [1]. The functions of transformer oil are to insulate, suppress corona discharge and arcing, and act as coolant [2,3]. The insulating oil properties should have high dielectric strength, thermal conductivity, chemical stability and able to handle high temperatures for extended periods. In order to ensure the secure operation of a transformer, testing procedures on dielectric quality of transformer oil have been conducted to fulfill the purpose of insulating as well as cooling. The testing procedures of new and 
aged oil samples produced results in oil resistivity, dissipation factor, conductivity, viscosity and breakdown voltage [4]. The conducted tests have been realized in accordance with the IEC standard test procedures which are IEC 60156, IEC 60245 and IEC 61125 [4]. In order to conduct the tests, insulating oils will be mixed in precise amounts to determine the breakdown voltage and the electrical field intensity of electro-convection. An oil sample is taken from the device under test to determine the insulating properties of the dielectric oil. The breakdown voltage of oil sample is measured on-site with the built-in electrodes, type of insulating oil and voltage injection level [5].

Transformer oil can be easily contaminated even during packaging, shifting and storing the transformer oil. Contaminants such as metal particles $[6,7]$ or cellulosic fiber residual can also be formed in the oil, especially for transformers with aged paper insulation [8]. During normal operation, non-uniform fields are present within the transformer. The contaminants tend to move towards high field regions due to dielectrophoresis (DEP) forces [9-11] and could form a bridge over a period of time. As shown in Figure 1, the cellulose particles that are seem to be behind the electrodes and in the middle of electrodes is the contaminants found in transformer oil. DEP phenomenon happened when a neutral body placed in an electric field become polarized and is equivalent to an electric dipole. One side of the dipole has an excess of positive charge and negative charge on the other pole. Under non-uniform electric field region, the DEP forces acting on the two ends do not balance and the particles move towards either high or low field region, as shown in Figure 1. The bridge may potentially act as a conducting path between two different potentials within the transformer structure, leading to partial discharges [12] or insulation failure [13, 14].

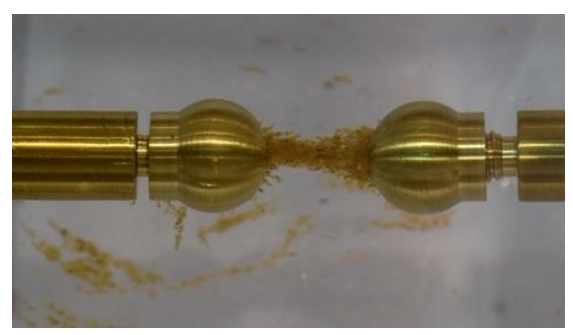

Figure 1. Example of electric field bridging formation in RGB image

As for now, there are limited literatures on the application of image processing technique to determine the pre-breakdown and breakdown condition. Thus, this work is conducted to analyze the electric field bridging images using digital image processing technique that has been applied in many studies involving mathematical morphology, neural networks, color image processing, feature extraction, image compression, image recognition and knowledge-based image analysis system [15, 16]. Digital image processing technique is chosen to visualize the image character and extract the important information [17] from the electric field bridging formation image. The findings in $[14,18]$ indicated that the bridging formation is depending on certain voltage value with some period of time. S. Mahmud et. al. [13, 14] describe the movements of cellulose particle were intensified at $15 \mathrm{kV}$ in which a thin complete bridge was made within $5 \mathrm{~s}$ and thickened up to $60 \mathrm{~s}$. This process indicates the pre-breakdown pattern that intiate a complete thickness of the bridge in 20 s which leads to breakdown pattern and for bigger size particle. The formation of bridging involved with the thickness of the bridge and the bond between the particles from smaller particles to bigger size particle [14].

The investigation of bridging pattern needs the involvement of feature extraction process. In machine learning, pattern recognition and in image processing, feature extraction begins with an initial set of measured data which is obtained from the measured object features. The features are intended to be informative and non-redundant in order to facilitate the subsequent learning and generalization step specifically in machine learning, which in same cases it is leading to human interpretations [19]. In this work, the bridging pattern is observed from the obtained recorded videos of electric field bridging formation to enhance researchers understanding on how the bridging is formed. The dataset of bridging images is created for pre-breakdown and breakdown condition. Image processing technique is then applied to extract important information and the feature descriptors are decided to measure the bridging pattern in pixels. The detail discussion on image processing technique, feature descriptors measurement and a brief introduction of classifier are described in Section 2. A feature descriptor or the combination of feature descriptors will result in the best classification process to determine the condition of the transformer oil. Three classification models [20] are used to measure the performance of classifier to classify the pre- and breakdown condition. The models are SVM, k-NN and RF classifier. The results of image segmentation outcome and 
the combination of feature descriptors in classifying the pre- and breakdown condition are described in Section 3. Finally, conclusion and future work is drawn in Section 4.

\section{RESEARCH METHOD}

In electric field bridging test $[5,11,13]$, voltage is injected on one side of the electrodes in which the voltage is increases by time. The images of electric field bridging formation are extracted from recorded video which were taken during data collection process. First, the images of electric field bridging are divided into two categories which are pre-breakdown and breakdown group. The categorization process as shown in Figure 2, is done manually by researchers through observation process. Figure 2 shows the examples of pre-breakdown, breakdown and post-breakdown images. Pre-breakdown condition is a condition captured before the breakdown happens. In initial state of observation in pre-breakdown condition, the cellulose particles seem scattered behind the electrodes. After certain period, the particles are forming an arc; single lines of unconnected bridge. The particles are found attracted to positive and negative charge on the electrode poles. This indicate the early sign of breakdown to happen. The formation of bridging is quickly form once the breakdown happened. The attraction of particles to the poles forms a thin to thicker bridging. The thicker bridging is caused by the increasing contaminant particles in oil, that provide a path for the current to conduct [21]. As the bridging formation increased, it caused current to explode. The explosion of current is seen in the video after certain period and certain voltage that demonstrates the testing oil is not safe to be used. Due to that, the particles started to drop down and lost contact from the electrode poles which is shown in post-breakdown images [14, 22].

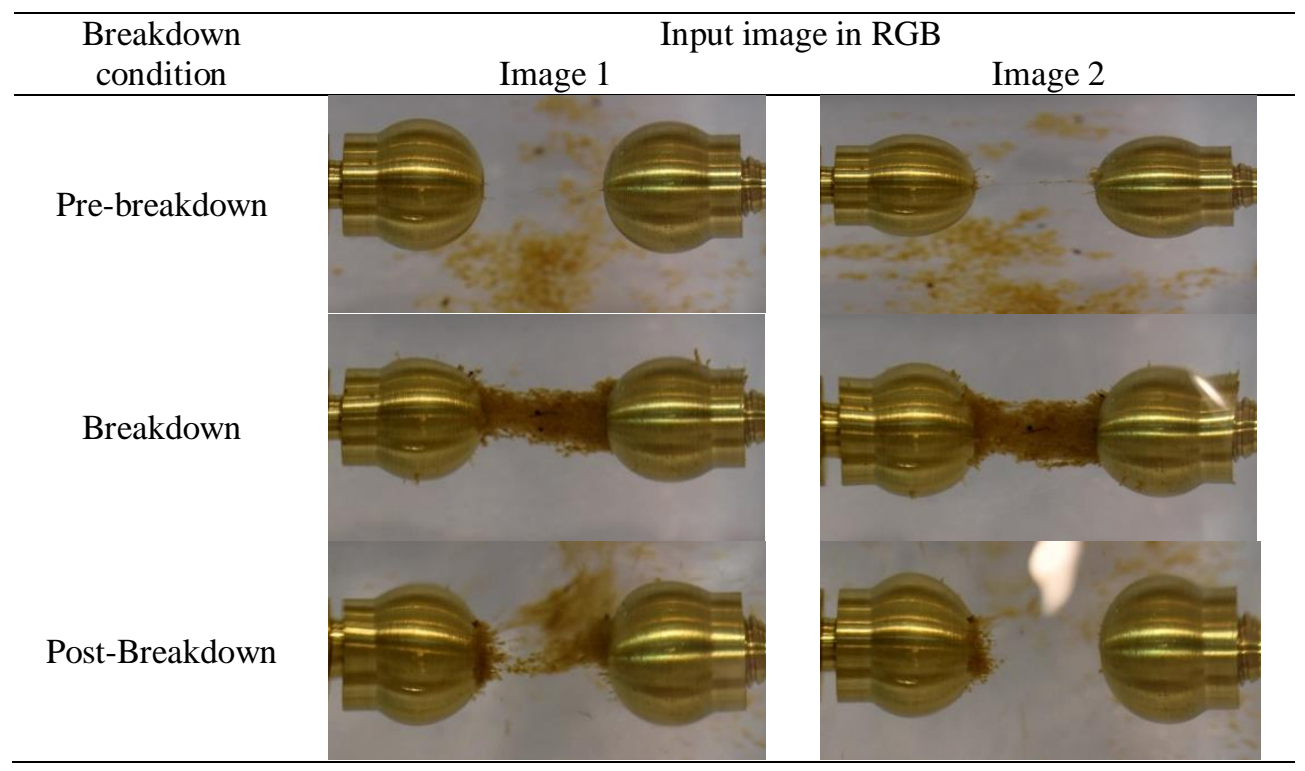

Figure 2. Input images of pre-breakdown, breakdown and post-breakdown condition

Second, the extracted images from the video are loaded into image processing algorithm in MATLAB. In this work, digital image processing technique is proposed in which this technique consist of three main stages which are pre-processing, feature extraction and classification [23, 24]. Pre-processing stage includes background subtraction and vertex marker as shown in Figure 3. Figure 3 shows the flow chart of pattern recognition technique in analyzing the breakdown condition of transformer oil based on electric field bridging pattern. Under pre-processing stage, the input image will go through two steps that are vertex marker and background subtraction. Figure 4 shows the red marker on the tip of electrodes. This marker is manually assigned by user. The vertex marker is an important step for the selection of measurement area named as region of interest (ROI). The markers will be the initial point of polygonal region of interest, roipoly in which the region of interest area will be extracted from the input image [25].

Figure 5 shows the cropped area of selected ROI by user. In background subtraction step, the electrodes, noise and white background will be removed in order to obtain the cropped area of selected ROI. Any features that lies inside this ROI will be evaluated and analyzed in feature extraction stage. 


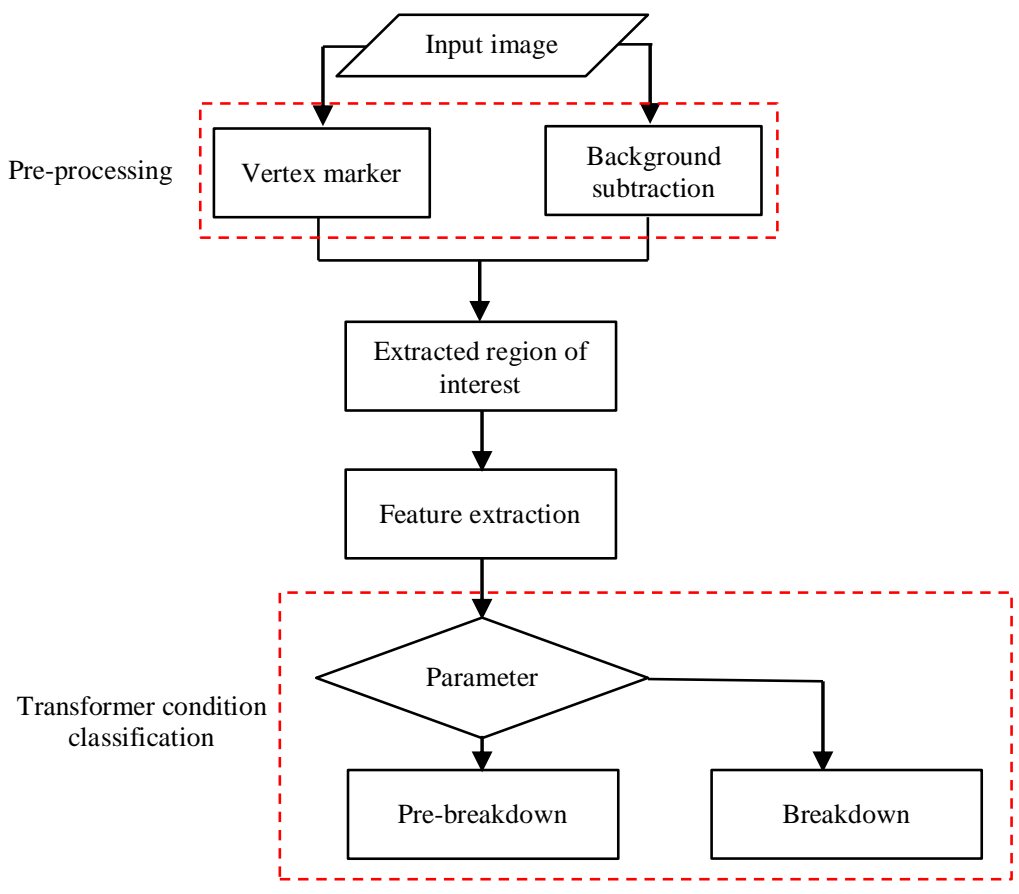

Figure 3. Flowchart of pattern recognition technique in analyzing the electric field bridging pattern

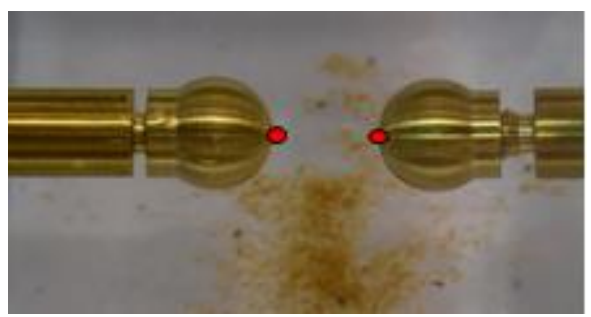

Figure 4. Vertex marker

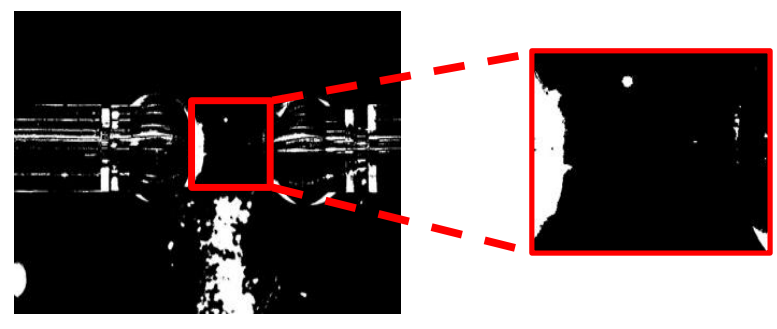

Figure 5. Measurement area, region of interest (ROI)

In the third stage, any object pixels that lie in the ROI will be measured and analyzed. Since the object pixels lie in the ROI is the closed bounded object, the pixel measurement technique is used to analyze the shape of the object or bridging. Feature extraction stage is used to interpret and capture the useful and important information. Based on the segmented bridging image, several feature descriptors can be determined such as area, minor-axis length and major-axis length [26]. Table 1 shows the definition of feature descriptors which can be obtained from the measurement of extracted object or electric field bridging. Three feature descriptors described in Table 1 are then used to classify the pre-breakdown and breakdown stages. In this work, the feature descriptors are determined using regionprops function in MATLAB. regionprops is used to measure properties of a selected image region in pixel count. In order to apply the regionprops function, the input image in RGB needs to be converted into a binary image. Then, bwboundaries function is called to trace the boundary of a selected region in an image. This labeled region will be represented in a two-dimensional array of non-negative integers [26]. The properties of regionprops such as area, minor-axis length and major-axis length are determined based on the labeled boundary of the selected region.

Table 1. Definition of feature descriptors

\begin{tabular}{cc}
\hline Feature Descriptor & Definition \\
\hline Area & The actual number of pixels in the ROI \\
Minor-axis length & The length of the minor axis of the biggest area (in pixels) \\
Major-axis length & The length of the major axis of the biggest area (in pixels) \\
\hline
\end{tabular}


The scope of this work is mainly on investigating the image characteristics of pre-breakdown and breakdown stages. However, the image characteristic of post-breakdown is not examined in this study since it does not provide any important information [5]. In other words, the transformer oil is identified as contaminated oil if the captured image of bridging formation shows the thicker bridging with higher pixel values. This characteristic is also representing the breakdown condition of the transformer. Finally, the combination of feature descriptors was tested and compared using support vector machine (SVM), k-nearest neighbor ( $\mathrm{k}-\mathrm{NN}$ ) and random forest (RF). A hold-out cross validation was applied to split the database into training and testing database, and to avoid overfitting. The training image database was fed into the classifier for training purpose. The trained classifier was then use to predict the testing image database without knowing the real feature of output values [27].

\section{RESULTS AND ANALYSIS}

In this section, the results of this research will be discussed in detail. The focus of this work is to investigate the pattern of electric field bridging of pre-breakdown and breakdown condition. For post-breakdown, it is not necessary to measure its pattern since it will not provide any important information [5]. In this work, 193 images were randomly chosen from the whole video frames where 102 images were the pre-breakdown images and 91 images were the breakdown images. These images were then be the input image to the image processing algorithm in order to segment the electric field bridging formation that was formed inside ROI. The output of this process was a binary segmented image as shown in Table 2. As presented in Table 2, there was a significant contrast between pre-breakdown and breakdown output images. In the output image of binarized image, black color represents image background, while white color represents the cellulose particles that formed from smaller particles (pre-breakdown) to bigger particle (breakdown) which is known as electric field bridging.

Table 2. Segmentation outcome of pre-breakdown and breakdown condition

Breakdown condition

In order to quantify the image pattern of pre-breakdown and breakdown, three feature descriptors were used as measurement tools. The feature descriptors will be measured based on pixels exist in the ROI. In this work, the proposed feature descriptors were area, minor-axis and major-axis length. The pixel values distribution of area, minor-axis and major-axis length for bridging formation pattern were shown in Figure 6 to Figure 8. Each feature descriptor results shows similar information pattern in which there is a significant pattern or distinct pixel range for pre-breakdown and breakdown condition. The pixel values of area, minor-axis and major-axis length in pre-breakdown condition is lower compare to breakdown condition. This is because in breakdown condition, the bridging formation has grown thicker by time [13, 28]. 
The thicker the bridging formation, the higher the pixel values. Under breakdown condition, images 103 to 176 show the increasing pattern of bridging thickness, while, for images 177 to 193, the bridging thickness show decreasing pattern. The decline of bridging pixel values represents the deterioration of transformer oil condition; a sign of post-breakdown pattern.

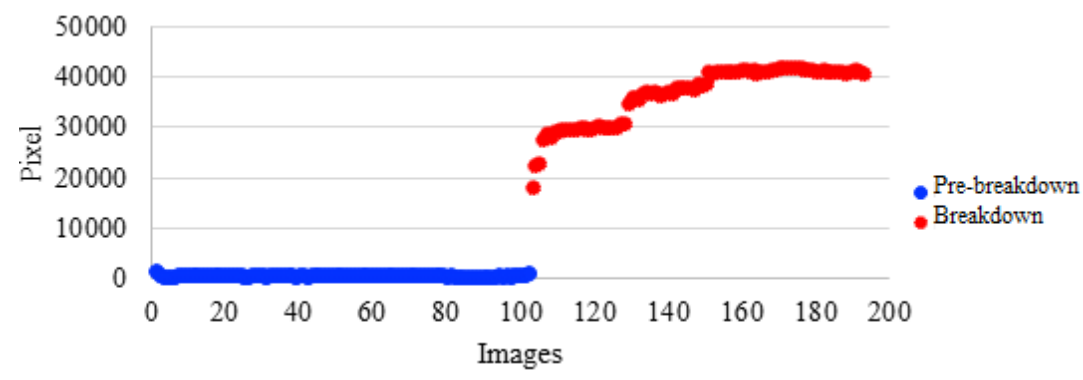

Figure 4. Area (in pixel) distribution of pre-breakdown and breakdown

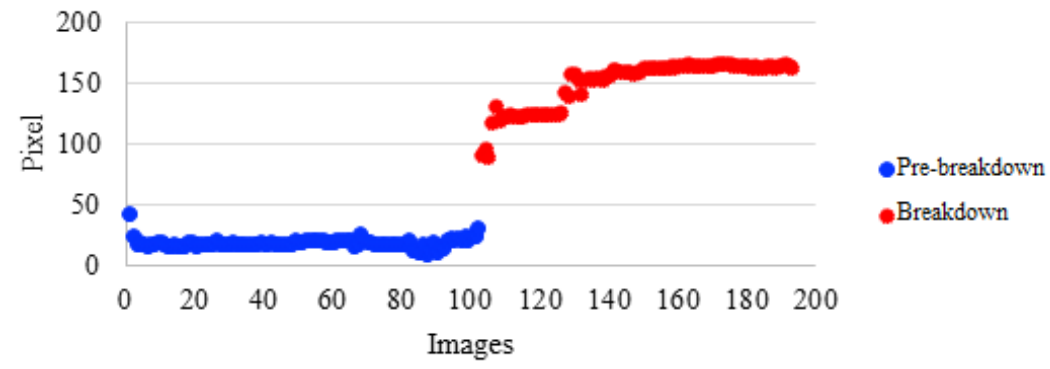

Figure 5. Minor-axis length (in pixel) distribution of pre-breakdown and breakdown

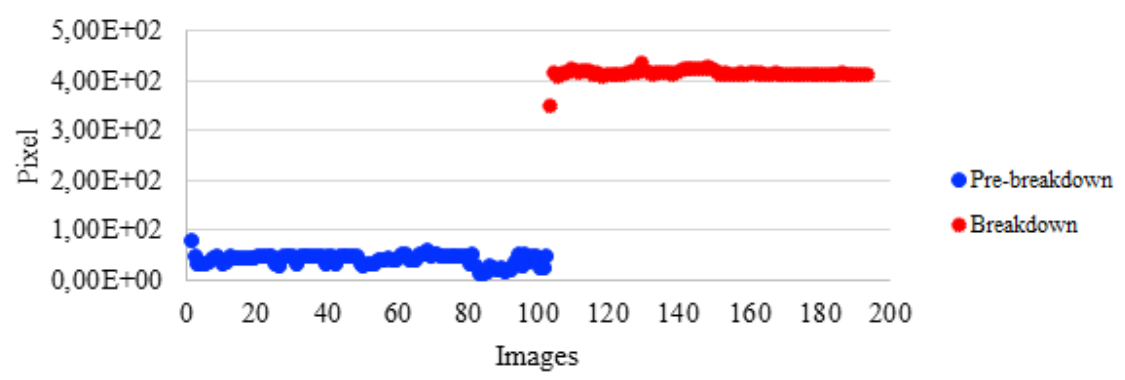

Figure 6. Major-axis length (in pixel) distribution of pre-breakdown and breakdown

Pattern classification techniques play an important role in the classification of pre-breakdown and breakdown condition. The process of pattern classification is simple in which after segmenting the bridging formation, a feature extraction and selection is performed in order to extract the relevant information from the region. In this work, three classification models are used to measure the performance of classifier to classify the pre- and breakdown condition. The models are SVM, k-NN and RF classifier. The result for each classifier algorithm is shown Figure 9. The outcome of each classifier is quite satisfying with the accuracy of 98\% for KNN, $99 \%$ for SVM and $98 \%$ for RF algorithm, tested using small number of data extracted from binarized segmented images [29]. The higher percentage of accuracy indicates that the combination of selected feature descriptors, area, minor-axis length and major-axis length are the perfect combination for classifying the pre- and breakdown condition. The proposed image processing and classification techniques successfully measured the pre-breakdown and breakdown patterns, and the features descriptors fed into the classifiers were capable to describe and classify the unique pattern between pre-breakdown and breakdown condition. 


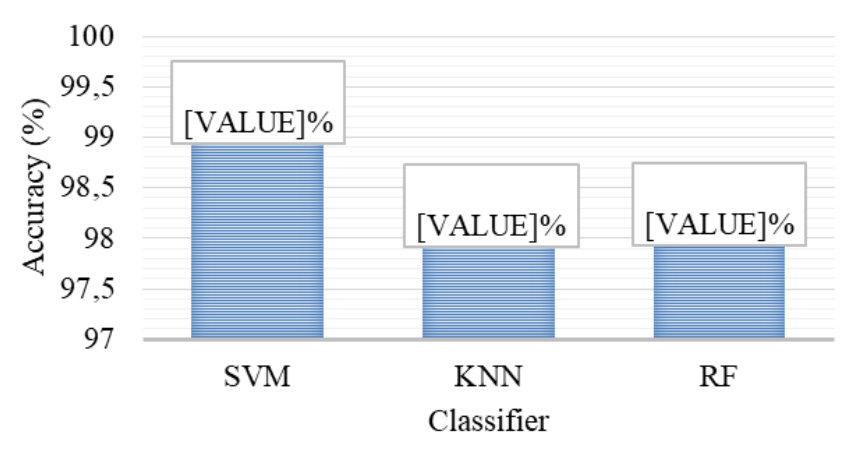

Figure 7. Accuracy of chosen classifier

\section{CONCLUSION}

The objective of this work is to investigate the pattern of electric field bridging formation of pre-breakdown to breakdown condition by using proposed image processing technique. It is found that the patterns of pre-breakdown and breakdown condition can be characterized by the feature descriptors such as area, minor-axis and major-axis length obtained from the binarized segmented image. For pre-breakdown condition, the pixel's value is considered lower compared to the pixel's value of breakdown condition due to thicker bridging pattern in breakdown condition. The thicker the bridging pattern, the higher the pixels value obtained. Three classification models were chosen which are SVM, $k$-NN and RF to validate the performance of the combined three feature descriptors in classifying the pre-breakdown and breakdown condition. From the results, it is found that SVM gives satisfying accuracy which is $99 \%$. In future work, further studies will be conducted to investigate the pattern of pre- and post-breakdown due to some similarity found in image pattern. Due to that, more feature descriptors will be identified to find a unique pattern between pre- and post-breakdown condition. As a conclusion, the proposed image processing technique can be used as a measurement tool with the proposed three feature descriptors that are area, minor-axis and major-axis length to monitor the condition of transformer oil.

\section{ACKNOWLEDGEMENTS}

The authors acknowledge Universiti Tenaga Nasional (UNITEN) for the UNIIG fund (Project Code: J510050843) given to support this project.

\section{REFERENCES}

[1] D. Amin, et al., "Recent Progress and Challenges in Transformer Oil Nanofluid Development: A Review on Thermal and Electrical Properties," IEEE Access, vol. 7, pp. 151422-151438, 2019.

[2] A. Kaur, et al., "Fault detection in power transformers using random neural networks," International Journal of Electrical and Computer Engineering, vol. 9, no. 1, pp. 78-84, 2019.

[3] S. Thakur, et al., "Experimental Investigation on Mustard Oil-Based Alumina Nanofluid Under Varying Temperature and Humid Condition," in Advances in Communication, Devices and Networking, pp. 133-142, 2018.

[4] J. Rouabeh, et al., "Studies of different types of insulating oils and their mixtures as an alternative to mineral oil for cooling power transformers," Heliyon, vol. 5, no. 3, pp. 1-19, 2019.

[5] M. M. Alharthi, et al., "Breakdown Voltage of the Transformer Oils under Certain Conditions," International Journal of Applied Engineering Research, vol. 13, no. 6, pp. 3810-3815, 2018.

[6] C. Pan, et al., "Partial discharge and breakdown characteristics of moving transformer oil contaminated by metallic particles," IEEE Transactions on Dielectrics and Electrical Insulation, vol. 25, no. 5, pp. 1774-1784, 2018.

[7] CIGRE.org, "Effect of particles on transformer dielectric strength," Techical Brochures, Work. Gr. 17 Study Committe 12, 2000.

[8] M. F. Rahman, et al., "Effect of Non-conducting Particle in Transformer Oil Partial Discharge Characteristics," in The 21st International Symposium on High Voltage Engineering, vol. 2, pp. 1014-1023, 2019.

[9] P. R. C. Gascoyne and J. Vykoukal, "Particle separation by dielectrophoresis," Electrophoresis, vol. 23, no. 13, pp. 1973-1983, 2002.

[10] S. Mahmud, et al., "Experimental studies of influence of different electrodes on bridging in contaminated transformer oil," IEEE Transaction on Dielectrics and Electrical Insulation, vol. 22, no. 5, pp. 2433-2441, 2015.

[11] S. Mahmud, et al., "Experimental Studies of influence of DC and AC Electric Fields on Bridging in Contaminated Transformer Oil," IEEE Transaction on Dielectrics and Electrical Insulation, vol. 22, no. 1, pp. 152-160, 2015. 
[12] Y. O. Shaker, "Detection of partial discharge acoustic emission in power transformer," International Journal of Electrical and Computer Engineering, vol. 9, no. 6, pp. 4573-4579, 2019.

[13] S. Mahmud, et al., "Bridging in contaminated transformer oil under DC and AC electric field," Journal of Physics: Conference Series, vol. 472, p. 012007, 2013.

[14] S. Mahmud, et al., "Bridging Phenomenon in Contaminated Transformer Oil," in 2012 IEEE International Conference on Condition Monitoring and Diagnosis, pp. 180-183, 2012.

[15] L. N. T. Alves and E. G. Costa, "Electrical Insulators Hydrophobicity Classification using Digital Image Processing," The 20th International Symposium on High Voltage Engineering, ISH Collection, 2017.

[16] S. H. Khaleefah, et al., "Review of local binary pattern operators in image feature extraction," Indonesian Journal of Electrical Engineering and Computer Science, vol. 19, no. 1, pp. 23-31, 2020.

[17] G. Kumar and P. K. Bahtia, "A detailed review of feature extraction in image processing systems," in Fourth International Conference on Advanced Computing \& Communication Technologies, pp. 5-12, 2014.

[18] M. H. S. Zainoddin, et al., "Investigation of the Dielectrophoresis Effect on the Electrical Performance of Dielectric Liquid," ARPN Journal of Engineering and Applied Sciences, vol. 12, no. 23, pp. 7003-7007, 2017.

[19] A. Smola and S. V. N. Vishwanathan, "Introduction to Machine Learning," Cambridge University Prss, UK, 2008.

[20] N. S. Zaini, et al., "Phishing detection system using machine learning classifiers," Indonesian Journal of Electrical Engineering and Computer Science, vol. 17, no. 3, pp. 1165-1171, 2020.

[21] G. Chen and M. H. Zuber, "Pre-breakdown Characteristics of Contaminated Power Transformer Oil," 2007 Annual Report-Conference on Electrical Insulation and Dielectric Phenomena, pp. 659-662, 2007.

[22] M. H. S. Zainoddin, et al., "Dielectrophoresis Effect of Dielectric Liquids with Suspended Cellulose Impurities under DC Electric Field," International Journal of Electrical and Computer Engineering, vol. 7, no. 6, pp. 3254-3261, 2017.

[23] S. Joshi and A. Kumar, "Feature extraction using DWT with application to offline signature identification," in Proceedings of the Fourth International Conference on Signal and Image Processing, India, pp. 285-294, 2012.

[24] A. Ibrahim, et al., "Characterization of cracking in pavement distress using image processing techniques and kNearest neighbour," Indonesian Journal of Electrical Engineering and Computer Science, vol. 14, no. 2, pp. 810-818, 2019.

[25] S. Wang, "An Adaptive Detection Algorithm for Small Targets in Digital Image," in Chinese Conference on Image and Graphis Technologies, pp. 333-339, 2015.

[26] N. B. A. Mustafa, et al., "Image processing of an agriculture produce: Determination of size and ripeness of a banana," in 2008 International Symposium on Information Technology, vol. 1, pp. 1-7, 2008.

[27] W. M. D. Wan Zaki, et al., "Automated pterygium detection method of anterior segment photographed images," Computer Methods and Programs in Biomedicine, vol. 154, pp. 71-78, 2017.

[28] M. H. S. Zainoddin, et al., "Effects of viscosity of ester oils and different sizes of suspended cellulose particles on bridging phenomenon under non-uniform DC electric field," in 2016 IEEE International Conference on Power and Energy (PECon), pp. 285-289, 2016.

[29] R. A. Nugrahaeni and K. Mutijarsa, "Comparative analysis of machine learning KNN, SVM, and random forests algorithm for facial expression classification," in 2016 International Seminar on Application for Technology of Information and Communication (ISemantic), pp. 163-168, 2016.

\section{BIOGRAPHIES OF AUTHORS}

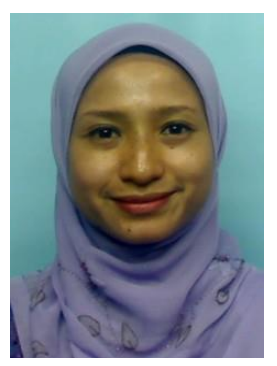

Nur Badariah Bt Ahmad Mustafa, received her PhD in Electrical, Electronics and Systems Engineering (2018) from Universiti Kebangsaan Malaysia (UKM), Master of Electrical Engineering (2010) and Bachelor of Electrical and Electronics Engineering (Hons.) (2007), both from Universiti Tenaga Nasional. She is a Senior Lecturer of Department of Electrical and Electronics, College of Engineering, Universiti Tenaga Nasional. She is a member of IEEE Signal Processing Society and graduate member of Board of Engineers Malaysia. Her research interest are on medical image processing, pattern and feature analysis and identification.

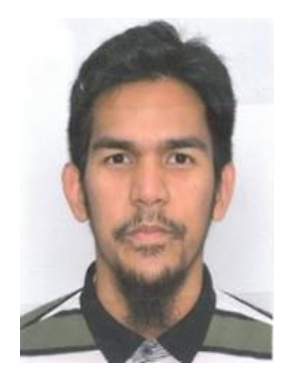

N. H. Nik Ali was born in K. Terengganu, Malaysia in 1990. He received the B.Eng. degrees in Electrical Power Engineering (First Class Honors) from the Universiti Tenaga Nasional (UNITEN), Malaysia in 2013. He received the Ph.D. degrees in Electronics and Electrical Engineering from the University of Southampton, UK in 2017. He worked as a Research Fellow in School of Electronics and Computer Science in University of Southampton, UK from 2018 to 2019. Currently, he is a Post-doctoral Researcher in Institute of Power Engineering (IPE) in UNITEN, Malaysia. He is also a graduate member of Board of Engineers Malaysia (BEM). His research interests are within the generic areas of applied signal processing. Within high voltage engineering this includes condition monitoring of high voltage cables and transformers, partial discharge measurement, HV insulation/dielectric materials, transformer rating analysis and applied signal processing. 


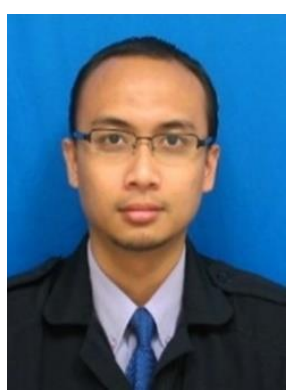

Hidayat Zainuddin received his Bachelor's degree in Electrical Engineering from Universiti Teknologi Malaysia in 2003. He then obtained his Master of Science degree in Electrical Power Engineering with Business from University of Strathclyde, Glasgow, in 2005. He received his Ph.D. degree from University of Southampton in 2013. He has served as an academic staff at Universiti Teknikal Malaysia Melaka (UTeM) since 2003 and currently, he is an Associate Professor at the Faculty of Electrical Engineering, UTeM. Administratively, he is the Deputy Dean (Student Development) since 2016 until present. His research mainly towards the sustainability of electrical power system which are associated with green technology, condition monitoring and failure analysis for high voltage transformers.

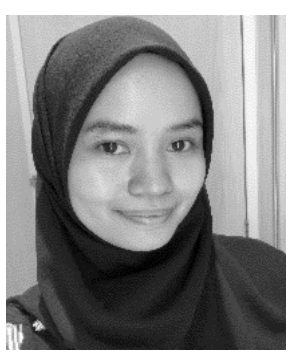

Marizuana Bt Mat Daud, received her PhD in Electrical, Electronics and Systems Engineering (2019) from Universiti Kebangsaan Malaysia (UKM), M.Sc. degree from University Technology PETRONAS (2014) and B.Eng. degree in Electrical and Electronics Engineering from University of Malaysia Pahang (2011). She is currently a consultant in MIMOS Berhad. Her research interest includes biomedical imaging, surveillance imaging, feature matching and feature calculation. She is the author a book titled with Pengesanan Penyakit Mata (Tunas, 2018).

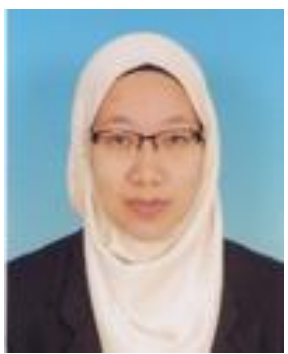

Farah Hani Nordin received her PhD in Engineering (2010) from Universiti Tenaga Nasional, MSc. in Control, Communication and Digital Signal Processing (2002) from University of Strathclyde and Bachelor in Electrical \& Electronic Engineering (Hons), from Universiti Tenaga Nasional in 2000. Farah Hani is an Associate Professor and currently holding the position of Deputy Dean (Student Affairs \& External Relations) under the College of Engineering, Universiti Tenaga Nasional (UNITEN). Previously, she has served as the Head of Electronics and Communication Engineering Department from 2014 until 2018 in College of Engineering, UNITEN. Farah Hani is a member of The Institution of Engineers Malaysia (IEM) and also registered as Professional Engineer under the Board of Engineers Malaysia. 